\title{
CLINICAL EVALUATION OF A UNIVERSAL ADHESIVE IN NON-CARIOUS CERVICAL LESIONS
}

by

\author{
Matthew A. Rouse
}

Submitted to the Graduate Faculty of the School of Dentistry in partial fulfillment of the requirements for the degree of Master of Science in Dentistry, Indiana University School of Dentistry, 2016. 
Thesis accepted by the faculty of the Departments of Cariology and Dentistry and Dental Public Health, Indiana University School of Dentistry, in partial fulfillment of the requirements for the degree of Master of Science in Dentistry.

Bruce Matis

Richard Jackson

Timothy Carlson

Jeffrey Platt

Chair of the Research Committee

N. Blaine Cook Program Director

Date 


\section{ACKNOWLEDGMENTS}

I thank God every morning for blessing me with outstanding people whose support and mentorship throughout this project allowed it to be successful.

To my wife, Kathryn, and daughters Nadia, Lucy, and Fiona, whose love, support, and encouragement refresh me personally and professionally every day, I give my sincerest love and appreciation.

I am overwhelmingly indebted to my mentors, Drs. Jeff Platt, Bruce Matis, Tim Carlson, Richard Jackson, and Michael Cochran. Your willingness to offer helpful suggestions and unrelenting support have been an immense blessing; I have learned more from you during my time in the Graduate Operative program at IUSD than I previously thought possible.

I wish to likewise thank my program director, Dr. Blaine Cook, who has been not only a mentor and constant resource, but also a role model, both personally and professionally; his experience and wisdom are truly unparalleled.

A very special thanks is deserved by the clinic coordinator of the Graduate Operative clinic, Judy Haines, who constantly displayed unwavering efforts in scheduling, advising, assisting, and other duties too numerous to mention in this short segment; she truly is the heart and soul of the Graduate Operative program. Finally, I wish to thank the United States Navy for the opportunity to study Operative and Preventive Dentistry at IUSD and to Ivoclar Vivadent for funding this study. 


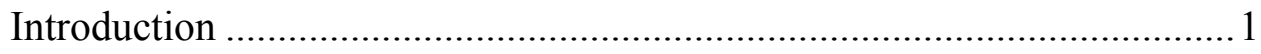

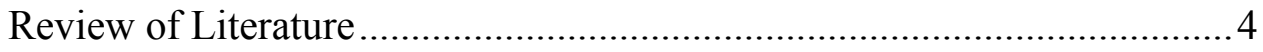

Methods and Materials ................................................................ 21

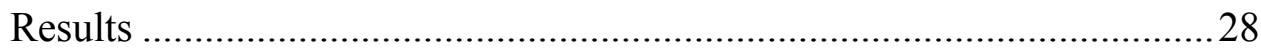

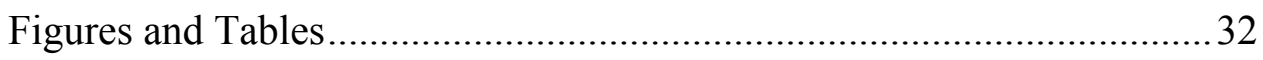

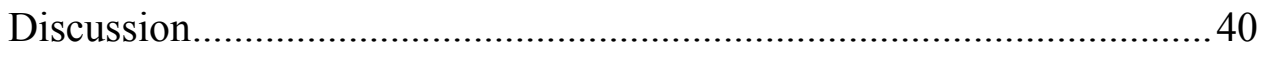

Summary and Conclusions ............................................................ 48

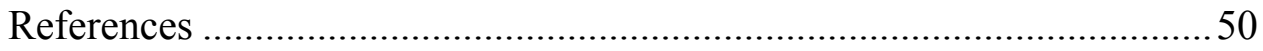

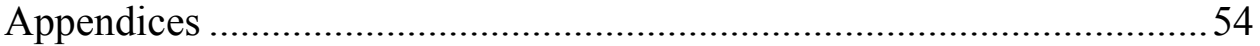

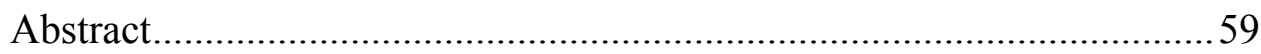

Curriculum Vitae 


\section{LIST OF ILLUSTRATIONS}

TABLE I Formulation of Adhese Universal...........................33

TABLE II Statistical analysis of faculty evaluator

calibration

TABLE III Pre-operative sensitivity rated from 0 to 10

by subjects.

TABLE IV 12-month post-operative sensitivity rated from

0 to 10 by subjects.

TABLE V Baseline, 6-month, and 12-month data comparing group differences for marginal adaptation, marginal discoloration, retention, and sensitivity 35

TABLE VI Marginal adaptation, marginal discoloration, retention, and sensitivity data for SelE and SfE groups compared at baseline, 6-months, and 12-months.

FIGURE 1 Pre-operative isolation of adjacent non-carious cervical lesion \#12-B

FIGURE 2 Post-operative photo of \#12-B following incremental placement of composite.

FIGURE 3 Non-carious cervical lesions \#20 and \#21 exhibit heavy dentin staining 38

FIGURE 4 Staining still shows through both restorations \#20 and \#21 after restoration with resin composite. 38

FIGURE 5 Restorations on teeth \#20 and \#21 at 6-month recall 39 

INTRODUCTION 
Non-carious cervical lesions (NCCLs) have been well documented in the dental literature for more than a century. ${ }^{1-2}$ Differing opinions exist concerning the etiology of NCCLs, but many agree that possible etiologies include abrasion, erosion, and abfraction, a term coined in $1991 .^{3-4}$ Although, some patients may not experience adverse effects from the presence of NCCLs, many experience sensitivity, ranging from mild to severe. The presence of NCCLs may also compromise the esthetics of the dentition.

For decades, resin adhesives have been used to restore non-carious cervical lesions for esthetics and/or patient comfort. Restoration of these lesions with bonded resin composite has been shown to be more effective, compared to no treatment or topical application of a desensitizing dentifrice. ${ }^{5}$ Since the advent of "fourth-generation" (also referred to as "3-bottle") resin bonding agents in the 1990 s, the primary focus in the improvement of resin adhesives has been simplifying the components, and thus the protocol for placement of resin composite restorations. New "universal adhesives" claim to simplify the process of bonding resin composites to tooth structure, while maintaining or exceeding the quality of previous adhesive systems.

Since dentin and enamel substrates are vastly different with respect to their composition and require different bonding protocols, some practitioners have advocated a "selective etch" procedure, in which the enamel and dentin are etched differently but may still be bonded using the same bonding agent. An in vitro study by Hanabusa et al. (2012) indicates that use of a multimode adhesive with selective etching of enamel with 
phosphoric acid provides better bonding efficacy than when the adhesive is used as a selfetch alone. ${ }^{6}$ Two in vivo studies ${ }^{7-8}$ also indicated a significantly improved performance of the selective enamel etch technique, though only one of the studies utilized a one-step universal adhesive. ${ }^{8}$ The other aforementioned study used a two-step bonding system comprised of a self-etch primer and separate bonding resin, as opposed to a one-step system. ${ }^{7}$ Other studies ${ }^{9-10}$ failed to demonstrate a significant difference between the two techniques. Since significant hydrolysis of the dentin-resin interface occurs after six months or even 12 months, ${ }^{11}$ a clinical trial of at least 18 -months' duration ${ }^{8}$ is indicated to more accurately depict the likelihood of long-term clinical success.

The purpose of this prospective clinical trial was to evaluate the efficacy of a newly formulated "universal" dental adhesive formulation (Adhese Universal, Ivoclar Vivadent) in non-carious cervical lesions in the permanent dentition using either a selfetch or selective-etch approach; the aim was to determine whether or not selective etching provided significantly improved retention, marginal adaptation, decreased sensitivity to a blast of air, and/or better resistance to enamel margin discoloration compared to self-etching over a 24-month period. This document contains data through 12 months of follow-up.

The null hypothesis was that there would be no statistically significant differences with respect to retention, marginal adaptation, or marginal discoloration of restorations placed with either the selective-etch or self-etch methods. The alternate hypothesis was that the restorations placed utilizing the selective-etch method would be superior in terms of retention and marginal adaptation, and they would have less marginal discoloration when compared to restorations placed with the self-etch method. 
REVIEW OF LITERATURE 


\section{ENAMEL BONDING}

Human enamel is approximately $96 \%$ inorganic hydroxyapatite crystal by weight (about $86 \%$ by volume), with the remaining proportion comprised of water and organic matrix. ${ }^{12}$ Hydroxyapatite crystals exist in the form of parallel rods, with other interrod crystals dispersed between the rods. These crystals are hexagonal in shape and their orientation directly affects the extent to which they are dissolved in the presence of acid; this is critically important in the context of resin bonding. ${ }^{13}$ Specifically, the ends of the rods must be exposed by mechanical preparation (i.e., beveling with either hand or rotary instruments) to ensure that acid-etching will effectively create the desired etched surface for resin bonding, which allows for a more intimate resin-enamel bond. ${ }^{14-15}$ Acid dissolution is possible, because hydroxyapatite crystals in dental enamel exist in an impure form with carbonate present in the lattice, making the enamel more susceptible to acid dissolution than would a perfect hydroxyapatite crystal. ${ }^{16}$ In fact, the acid dissolution of enamel, discovered by Dr. Michael Buonocore in 1955, eventually led to the acidetching protocols used today. Although Buonocore's original protocol of $85 \%$ phosphoric acid applied to enamel for 60 seconds has been significantly modified, his discovery laid the framework for modern enamel etching and bonding. ${ }^{17}$ When enamel is etched by acid, pores are created, and the surface area may be increased by a factor of 20 . Not only does acid create pores and microroughness in enamel, but acid-etching also enhances the surface energy of the enamel. Before polymerization of the monomer, the increase in 
surface energy allows the resin monomer to infiltrate and form resin tags in enamel, significantly increasing micromechanical retention. ${ }^{18-19}$

\section{DENTIN BONDING}

Improvements in dentin bonding have occurred over the last 30 years but resindentin bonding still falls short of the reliability and longevity of resin-enamel bonding. While enamel is about $86 \%$ inorganic material (by volume), dentin is only about $45-50 \%$ inorganic hydroxyapatite and 30\% organic material and dentin contains significantly more water than enamel (25\% versus $1-4 \%) .{ }^{20}$ These differences in morphology cause the two substrates to interact very differently with resin adhesives. After etching, enamel can be dried thoroughly until a frosty, chalky appearance is observed, indicating it is ready to receive the resin-bonding agent. Bonding to dentin is much more technique-sensitive. While some type of acid (commonly phosphoric) is still applied to demineralize the dentin's collagen fibers to allow the resin to interlock with the fibers, this substrate must be managed with precision to achieve the strongest, most durable bond, particularly in terms of drying time, since the composition of dentin is much greater in organic matrix and water than in enamel. The relative terms "dry-, moist-," and "wet-bonding" have been used to attempt to describe the extent to which the dentin should be dried. If too much residual water is present when the bonding agent is applied, the bonding agent is diluted and may not polymerize appropriately, leading to significant marginal leakage; conversely, over-drying the dentin causes collapse of the collagen matrix, which significantly inhibits resin penetration of the matrix. ${ }^{21}$ As a result, there is a somewhat narrow window of moisture that is conducive to optimal dentin bonding. 
In addition to moisture, degradation of the collagen may also threaten the longevity of the resin-dentin bond. Cholesterol esterase, an enzyme found in human saliva, has been shown to degrade collagen in vitro, ${ }^{22}$ as have matrix metalloproteinases (MMPs). Degradation of collagen results in a weakened resin bond. ${ }^{11}$ In addition to human enzymes, bacterial collagenase enzymes are also capable of degrading collagen, also resulting in a weakening of the resin-dentin bond. ${ }^{23}$ Not only do different enzymes degrade collagen at the resin-dentin interface, hydrolysis of the resin itself has also been observed when stored in water. ${ }^{24}$ With these obstacles to dentin bonding, the task of creating a material that can reliably bond to dentin has gone through significant evolution over the last 30 years. The different classes of dentin-bonding agents will be discussed in further detail in a subsequent section.

\section{NON-CARIOUS CERVICAL LESIONS}

A non-carious cervical lesion (NCCL) can be defined as the "progressive loss of tooth structure at the CEJ." ${ }^{25}$ As the name implies, these lesions are not due to demineralization from bacterial byproducts, but rather they are multifactorial in nature and their etiologies may be a combination of chemical and/or mechanical processes. These processes include abrasion, erosion, and abfraction. Though there is some disagreement regarding the exact mechanisms of structure loss in NCCLs, the different mechanisms are generally agreed upon by most dentists. ${ }^{4}{ }^{26}$ NCCLs are generally observed in one of two shapes: saucer-shaped lesions, which are typically smooth and rounded, and wedge-shaped lesions, which form a more acute angle at the axial wall of the lesion. It has been implied that saucer-shaped lesions are usually erosive in etiology 
and are caused by dietary and/or gastric acids, while abfraction and abrasion lesions typically cause a wedge-shaped lesion. Abfraction lesions are thought to be due to nonaxial occlusal stresses, which cause subclinical microfractures of tooth structure, though studies have failed to support this theory. ${ }^{26}$ Abrasive lesions are thought to be primarily due to toothbrush bristles and abrasives found in toothpastes. ${ }^{4}$ The treatment for an advanced NCCL, where significant tooth structure has been lost in an axial direction, is restoration of lost tooth structure, regardless of etiology. Many materials are routinely used for the restoration of NCCLs. One of the most common materials used is resin composite. The successful use of resin composite to restore NCCLs has been well established in scientific dental literature, with studies reporting clinical success rates of $91-100 \%$ after five years. ${ }^{27-28}$

One possible dentin response in NCCLs is the formation of hypercalcified, or sclerotic dentin. The superficial sclerotic dentin layer contains denatured, non-crosslinked collagen. The occlusion of dentin tubules, as observed in sclerotic dentin, likely occurs through a complex process of demineralization (via bacterial acids) and subsequent remineralization. This remineralization process entombs bacteria to form a mineralized bacteria matrix and is likely enhanced by the presence of fluoride in the saliva. ${ }^{29}$ In vitro studies have demonstrated that the acids contained in many self-etching adhesive systems are incapable of penetrating this thin sclerotic layer and bond strengths are therefore compromised in comparison to dentin without the presence of occluded tubules; mechanical preparation or extended etching with strong acids may remove this sclerotic layer and facilitate better bond strengths. ${ }^{29-30}$ Even with chemical preparation, such as 
increased etching times and stronger acids, the rod-like plugs that obliterate the dentin tubules are not removed and resin tags are minimal or absent. ${ }^{31}$

\section{CLASSIFICATION AND OVERVIEW OF ADHESIVE SYSTEMS}

Adhesives may be classified by one of four methods: solvent type and concentration (not commonly used), mechanism of smear layer modification/removal, generation, or number of clinical steps. Formerly, the most common method of classifying adhesive systems was by generation. Ordered chronologically by introduction into the dental materials market, the products within each generation possess similar characteristics that differ significantly between generations. There are currently eight generations of adhesive systems. Due to the overlap in terminology, generations will be the primary method of classification used later to describe the different adhesive systems, but the method of smear layer removal and the number of clinical steps may also be used for clarification purposes.

There are two ways to treat the smear layer; both are important to understand before discussing the different adhesive systems. Etch-and-rinse (also known as "total etch," since it totally removes the smear layer) systems use a strong acid (typically phosphoric acid between $35-40 \%$ ) to etch the tooth substrate for a specified period of time before completely rinsing and drying the tooth to some degree before subsequent application of the primer. In contrast, self-etching (also known as "etch-and-dry") systems do not remove the smear layer completely; instead, they dissolve it so that the adhesive system can still penetrate enamel and dentin.

Classifying adhesives by the number of clinical steps is probably the most 
common and simple method used, although classifying by the number of steps alone leaves out critical information regarding the system, such as which components are combined and what the means of the smear layer treatment is. For this reason, the number of clinical steps is usually used, along with the method of smear layer treatment; for example, one may refer to a certain adhesive system as a "sixth-generation adhesive" or a "two-step, self-etch adhesive." In this example, the meaning of "two-step" is obvious and "self-etch" refers to the mechanism of smear layer modification. This gives far more information about the adhesive system than the generation alone.

The first generation of adhesives was developed in the 1960s based on a "surface active comononer," NPG-GMA, which theoretically formed a water resistant bond to calcium in tooth structure. Unfortunately, these systems showed poor clinical performance, with bond strengths of only about 2-3 megapascals (MPa). The second generation of adhesives came in the 1970s and was similar to the first generation with respect to the use of hydrophobic resins and the lack of smear layer removal. These systems used either phosphate monomers or 4-methacryloyloxyethy trimellitate anhydride (4-META), which was developed in Japan in 1979 but not readily available in the United States for about eight years. ${ }^{32}$ Like first-generation adhesives, the secondgeneration systems had poor bond strengths of only about 2-4 MPa to dentin (10-20 MPa to enamel). A giant leap in adhesive technology came with the third generation of adhesives when acids began to be used to etch/demineralize dentin (i.e., etch-and-rinse or total etch), allowing for better penetration into dentin due to dissolution and removal of the smear layer produced during preparation. Improved wetting, penetration into dentin tubules, and the formation of what is now known as a "hybrid layer" was also due to the 
incorporation of bifunctional primer molecules, which had a hydrophilic component and a hydrophobic component. ${ }^{33}$ With the improvement in technology came improved bond strengths of up to $13 \mathrm{MPa}$ to dentin and 10-30 MPa to enamel, representing a significant increase in strength from the second generation. The fourth generation of adhesive systems is considered the "gold standard" even today. This generation, referred to as three-step etch-and-rinse, introduced the concept of "wet bonding," which referred to etching, rinsing, and applying the primer to moist dentin. This ensured the patency of dentinal tubules in contrast to the collapsed, relatively impenetrable collagen network observed with desiccated dentin. The fourth-generation adhesives consist of a conditioner to remove the smear layer (commonly phosphoric acid), a bifunctional primer molecule, and a resin adhesive. Two of the adhesives from the fourth generation, Optibond FL (Kerr) and Scotchbond Multipurpose (3M ESPE), are still considered by many to be the best adhesives ever made available. Fourth-generation adhesive systems boast bond strengths of up to $13-80 \mathrm{MPa}$ to dentin. ${ }^{34-35}$ The fourth generation of adhesives brought about dentin bonding strengths comparable to enamel and, as a result, development of new adhesive systems then focused on simplifying the three-step, technique-sensitive protocol into something faster and easier without drastically sacrificing clinical performance. The fifth-generation adhesives still utilize a separate etchant/conditioner but combine the primer and bonding agent into one bottle (e.g., "one-bottle etch and rinse" or "two-step etch and rinse" systems), typically in an acetone solvent. Although initial bond strengths seem to be comparable to their fourth-generation counterparts, studies indicate that fifth-generation systems may be inferior when measured over a longer term. The most likely reason for this observation is the combination of the hydrophilic primer and 
hydrophobic ionic resin in one container; when the two components are combined and applied simultaneously, the result is a hybrid layer without a distinct hydrophobic resin layer, since the bonding interface may have hydrophilic molecules at the adhesive-resin interface. This causes the adhesive layer to function as a semipermeable membrane, allowing for the incorporation of water and subsequent hydrolysis of the hybrid layer. ${ }^{36}$ This compromised longevity of the bond created by fifth-generation adhesives may also be due to the high volume of solvent (up to $50 \%$ ). When the solvent is not completely evaporated, incomplete polymerization results. Separation of the primer and adhesive phases in the container is another concern; this could cause application of the components in incorrect proportions. Sixth-generation adhesives, like the fifth generation, are designed to simplify the application process through combining components, but these adhesive systems are classified as "two-step, self-etching," rather than "etch-and-rinse," because the etching agent is combined with the primer and is not rinsed off, but rather air-thinned (to evaporate solvent) and polymerized. Instead of combining the bifunctional primer molecule with the hydrophobic resin adhesive, the resin adhesive is a separate component, applied and polymerized only after polymerization of the self-etching primer. Unlike fifth-generation adhesives, this allows for complete coating of the substrate with hydrophobic resin, thereby creating a membrane that does not readily allow for the passage of water. Since there is no rinsing after application of the etchant and primer, the smear layer is not removed but rather demineralized (to allow for resin penetration) and incorporated into the hybrid layer. This alleviates the problem of over-etching, which occurs when an etching agent demineralizes substrate and is then rinsed away, leaving voids greater than the few micrometers that the adhesive is capable of penetrating. This 
over-etching, which may occur with total-etch systems, is mainly responsible for the presence of exposed collagen fibrils that lead to the degradation of collagen and subsequent failure of the adhesive interface. ${ }^{36}$ Along with fourth-generation systems, sixth-generation adhesives provide the highest performance of all current adhesive systems. A criticism of these systems, however, is that the weak acids used as etching agents may not be sufficient to demineralize enamel to an adequate degree in contrast to etch-and-rinse systems. ${ }^{33}$ Self-etching primers produce a shallower etching of enamel, which is not as retentive; strong acids with a $\mathrm{pH}<1$ must be used to produce an enameletching pattern similar to total-etch systems. ${ }^{37}$ Sixth-generation systems utilizing a mild etching agent do not produce the commonly referenced frosty appearance and have shown unsatisfactory enamel etching when compared to phosphoric acid. ${ }^{37}$ The problem with using strong acids with etch-and-dry adhesives is that strong acids are not buffered by dentin hydroxyapatite, as described earlier, and the acid will etch continuously and not be polymerized completely. ${ }^{38}$ The compromised bonding to enamel is typically preferred and most sixth-generation systems do not utilize strong acids. As fifth- and sixthgeneration systems simplified three- into two-step applications, the seventh- and eighthgeneration of adhesives simplified application even further by making adhesive application a one-step process. Seventh-generation systems are classified as "one-step" adhesives, although they come in multiple bottles, because they are combined just prior to use and applied as a single substance. Conversely, eighth-generation systems, or "allin-one" systems, are packaged with all components in a single bottle. When compared to multi-step adhesives, these one-step self-etch systems can certainly be applied faster; however, studies have not only shown lower immediate bond strengths but also poor 
durability compared to multi-step systems. Additional issues include increased water sorption, phase separation, and increased nanoleakage.

Universal adhesives are the most recent classification of adhesive systems to emerge in the dental materials market. Unlike other adhesive systems that make more conservative claims with regard to their indications, most universal adhesives available today claim to be indicated for most or all of the following applications: direct-placed light-curing composite and compomer restorations; direct-placed core build-ups with light-, self-, and dual-curing composites; repair of fractured composite and compomer restorations; adhesive cementation of ceramic or metallic indirect restorations with lightand dual-curing luting composites (due to the incorporation of methacrylated phosphoric acid esters discussed later ${ }^{39}$ ); sealing of prepared tooth surfaces before temporary or permanent cementation of indirect restorations; and desensitization of hypersensitive cervical areas. Universal adhesives are also indicated for use with a self-etch technique, a selective-enamel-etch technique, or an etch-and-rinse technique.

\section{SELECTIVE ENAMEL ETCHING}

The inability of self-etch bonding agents to adequately etch enamel due to their decreased acidity brought about the idea of "selective enamel etching," which calls for the application of a strong acid (37\% phosphoric acid gel) to enamel, while intentionally avoiding dentin. By following this protocol, enamel would be etched and rinsed just like the first clinical step for bonding with a fourth- or fifth-generation adhesive. Following the rinsing and drying of enamel, the self-etching adhesive would be applied to both enamel and dentin; the enamel would then receive the primer and adhesive and the mild 
acid contained in the self-etch adhesive would adequately condition, prime, and bond to dentin without over-etching. An in vitro study tested this theory in 2009 by comparing the enamel bond strengths achieved by sixth-, seventh-, and eighth-generation bonding systems (with and without selective enamel etching with phosphoric acid before application of the self-etching primer or adhesive) versus a fourth-generation etch-andrinse system. Without pre-etching, enamel bond strengths were significantly lower for all self-etch systems. When enamel was pre-etched, all four self-etch systems achieved enamel bond strengths comparable to the etch-and-rinse system and were significantly greater than bond strengths without pre-etching. ${ }^{40}$ These results were also tested in a fiveyear randomized clinical trial published in 2007 by Peumans, et al., in which a sixthgeneration bonding agent with mild acid was applied with (experimental group) and without (control group) selective enamel etching. ${ }^{27}$ The authors concluded that, while "additional etching of the enamel cavity margins resulted in an improved marginal adaptation on the enamel side...this was not critical for the overall clinical performance of the restorations." The group later published eight-year results that showed a statistically significant difference in superficial marginal discoloration, but the positive effect of selective enamel etching even at eight-years still appeared to be small and did not affect the overall clinical success of restorations. ${ }^{10}$ In 2014, Perdigão, et al. published 18-month results of a randomized, double-blinded clinical trial utilizing a universal adhesive system (Scotchbond Universal, 3M ESPE). This study showed that, after 18 months, the self-etch approach yielded a significantly greater number of restorations with marginal discrepancy greater than $30 \%$ of the bonded interface. Although both products are termed "universal," there is some ambiguity with this term in the dental materials 
market. While Scotchbond Universal is very similar to Adhese Universal, it makes the broader claim that it may be used for all indirect restorations; Adhese Universal claims to be indicated only where light can reach, limiting its indirect applications and warranting further investigation. Still, other adhesives may use the term "multi-mode," meaning they are appropriate for self- and selective-etch indications but not indirect applications.

\section{CHEMISTRY AND COMPOSITION OF IVOCLAR ADHESE UNIVERSAL}

Adhese Universal is composed of $67 \%$ monomers that include 2-hydroxyethyl methacrylate (HEMA), bisphenol A glycerolate dimethacrylate (Bis-GMA), decandiol dimethacrylate (DDDMA), methacrylated phosphoric acid ester, and methacrylated polyacrylic acid. First, HEMA functions as a "primer" or "wetting agent" due to the fact that it is completely miscible in water (as found in dentin tubules) and improves penetration of the adhesive system. Although studies have shown that HEMA alone does not necessarily function well on a moist substrate, HEMA coupled with a polyalkenoic copolymer (like methacrylated polyacrylic acid in the case of Adhese Universal) does indeed perform well in a moist environment. ${ }^{33}$ Bis-GMA is the major monomer in most resin-based dental materials; it is much less hydrophilic than HEMA, absorbing only about $3 \%$ of water after polymerization; ${ }^{41}$ although this property is undesirable for penetration of moist dentin tubules, it is advantageous for resistance against hydrolysis of the resin-dentin interface. This hydrophobic component is also the molecule that interacts in bonding to the subsequently placed resin composite material. DDDMA is also a hydrophobic molecule that has the characteristics of flexibility, increased wetting, and rapid polymerization. The methacrylated acids lower the $\mathrm{pH}$ of the adhesive and dissolve 
the smear layer, while also allowing the monomers to penetrate the enamel and dentin tubules. As the mineral component of the bonding substrate dissolves, free minerals function as a buffer to limit depth of penetration of the adhesive; therefore, the dentindissolving/etching ability of the monomer decreases as the depth of penetration into dentin increases. ${ }^{42}$ Specifically, methacrylated phosphoric acid esters, such as MDP, have a high affinity for enamel, dentin, metals, and zirconia, increasing the strength of the adhesive interface. ${ }^{39,43}$ Incorporation of these esters also renders the bonding interface less susceptible to degradation. Adhese Universal contains 4.0\% (by weight) fumed silica, which is the filler particle. The addition of filler particles is a somewhat complex concept, because over- or under-loading the adhesive with fillers can be detrimental to performance. An unfilled resin matrix exhibits volumetric contraction that may be as high as $10 \%$. This shrinkage causes stress at the adhesive interface, which could lead to debonding. ${ }^{44}$ Conversely, over-loading an adhesive with filler particles, while decreasing polymerization shrinkage (and the stresses that accompany it), would increase the viscosity of the adhesive and inhibit its wetting ability (e.g., the ability to penetrate dentin tubules). Because of the viscosity of monomers, especially Bis-GMA, a solvent is used to carry the monomer to the substrate and aid in wetting and penetration. A combination of ethanol and water constitutes $25 \%$ (by weight) of Adhese Universal. Ethanol is a commonly used solvent, because of its volatility and ability to displace water from the dentin surface and collagen network. Water is commonly found in self-etching adhesives, because it allows for dissociation of the acid monomers (release of a proton from the acid), which causes etching of the enamel and dentin substrates. ${ }^{45}$ Any residual unevaporated solvent, however, will act as a plasticizer, since it will not polymerize; this 
will weaken the physical properties of the polymerized resin network. This problem is more pronounced with water than other, more volatile solvents. Adhese Universal also contains two tertiary amines that serve as reducing agents (ethyl p-dimethylaminobenzoate and dimethylamino ethylmethacrylate), and the photoinitiator, camphorquinone (CQ). These compounds make up 3.8\% (by weight) of the adhesive. When visible, blue light with a wavelength of $\sim 470 \mathrm{~nm}$ reacts with camphorquinone, causing the camphorquinone molecules to become excited; in this state, the CQ molecules interact and actually break the Carbon-Carbon double bonds $(\mathrm{C}=\mathrm{C})$ present in the monomers. When these $\mathrm{C}=\mathrm{C}$ bonds break, monomer molecules form single $\mathrm{C}$ - $\mathrm{C}$ bonds with one another, creating a polymer network due to this so called "addition" reaction. This reaction is the cause of the volumetric shrinkage mentioned previously in reference to filler content. The final components of Adhese Universal are butylhydroxytoluene (BHT) and monomethyl ether hydroquinone (MEHQ), which are stabilizers/inhibitors. The combined weight of these molecules is only $0.1 \%$, since they are not part of the actual polymerization/bonding process. Conversely, they are present for the exact opposite reason; BHT and MEHQ act to "scavenge free radicals originating from prematurely reacted initiators. ${ }^{, 46}$ When adhesives are stored, especially at elevated temperatures, the monomers may begin to polymerize, which would render the adhesive useless for application. These molecules, even in such small amounts, are critical to ensuring that the adhesive is not polymerized until the desired time. While BHT is generally used for more hydrophobic resins, MEHQ is a frequent choice for more hydrophilic resins. The likely reason for the incorporation of both into Adhese is the presence of both hydrophilic and hydrophobic components. As of 2007, when the most recent data became available, 
questions of biocompatibility still exist with both these compounds, as both wash out of polymerized resin. $^{46}$ 
MATERIALS AND METHODS 


\section{ENROLLMENT/RANDOMIZATION}

A total of 33 subjects, ranging in age from 20 to 75 years, were recruited for this study, with the goal of roughly one-third each in the following age groups: $20-39,40-59$, and $\geq 60$. The study population was targeted to be comparable in terms of the ratio of men to women. Subjects were selected who met the inclusion/exclusion criteria (Appendix 1). The clinical design used each technique (self-etch and selective etch \& rinse, abbreviated SfE and SelE, respectively) in each of 33 subjects. Each subject had at least two teeth selected for inclusion in the study. The first tooth to be treated was randomly assigned to one of the two treatment groups using a randomization table. The second tooth was placed in the second group. If a third tooth was included, a randomization table determined which treatment it received.

\section{SUBJECT RECRUITMENT}

Subjects for this investigation were selected from patients of record at Indiana University School of Dentistry Clinics who met the inclusion/exclusion criteria. Dental students, dental hygiene students, and faculty were briefed on the study criteria to assist in identifying potential subjects. Subjects who called via telephone were guided through the IRB approved phone-screening document (Appendix 2). 


\section{CONSENT}

The consent process was completed by the student investigator. Potential subjects attended a screening visit in which they were given the IRB-approved informed consent and authorization (IRB protocol \#1403626552) for the release of health information for research form. Upon reading the documents, the subjects were asked if they had any questions. The purpose, procedures, risks, and benefits of the study were reviewed with each subject. The subjects signed and dated the consent and authorization forms after having their questions answered. Each person who completed the consenting process with the subject also signed and dated the consent. Copies of both documents were given to the subject.

\section{STUDY SCREENING PROCEDURES}

After informed consent had been obtained, the Principal Investigator or Student Investigator reviewed the subject's medical record to ensure accuracy (Axium files), asked the inclusion/exclusion questions, and performed an exam of the mouth. If the subject qualified to participate, the teeth involved in the research study were identified and randomized by the student investigator according to the randomization table.

\section{RESTORATIVE PROCEDURES}

Prior to placement of the restorations, the following information was recorded by the student investigator (Appendix 3): evidence of sclerosis (as determined by glossy appearance and glassy feel when examined with an explorer [the percentage was visually estimated]), lesion morphology (predominantly saucer-shaped or predominantly notch- 
shaped), evidence and location of occlusal facets, and pre-operative sensitivity to a blast

of air (from approximately $1 \mathrm{~cm}$ for $1 \mathrm{~s}^{47}$ ) using a numeric pain scale ranging from 0 to 10 as a standard index (Appendix 4).

Intraoral digital photographs were taken by the student investigator using a Canon Rebel T3 camera with a Tamron 90 mm macro lens (Canon USA, Melville, NY). Restorative procedures were performed by the student investigator on each tooth following standard of care procedures for a Class V restoration. All teeth were cleaned with pumice and a prophy brush for approximately 10 seconds prior to treatment. The subject read and signed the Indiana University School of Dentistry's consent for treatment. Standard of care treatment included: no mechanical preparation or beveling of the tooth surface for either treatment group, local anesthetic was offered to each subject and its use was guided by patient response, and rubber dam isolation using a 212 clamp was used for each restoration (Figure 1). Thirty of 33 subjects requested anesthetic.

\section{SELF-ETCH PLACEMENT}

The universal adhesive (Adhese Universal; Ivoclar Vivadent, Lot \#T02457) was applied by the student investigator utilizing the following instructions from the manufacturer:

Starting with enamel, thoroughly coat the tooth surfaces to be treated with Adhese Universal. The adhesive must be scrubbed into the tooth for at least 20 seconds. This time must not be shortened. Disperse Adhese Universal with oil- and moisture-free compressed air until a glossy, immobile film layer results. Light-cure Adhese Universal for 10 seconds using a light intensity of $\geq 500 \mathrm{~mW} / \mathrm{cm}^{2}$. 
For lesions greater than $2 \mathrm{~mm}$ in any dimension, incremental placement of Tetric EvoCeram composite (Ivoclar Vivadent) was utilized, with the first increment being placed against enamel (Figures 1 and 2). Instructions are described below:

Apply Tetric EvoCeram in increments of max $2 \mathrm{~mm}$ and adapt with a suitable instrument. Sufficient exposure to light prevents incomplete polymerization. Remove excess material with suitable finishers or fine diamonds after polymerization. Use silicone polishers, as well as polishing disks and strips, to polish the restoration to a durable, high gloss.

Each increment was light-cured using the intensity and duration prescribed by the manufacturer ( $>500 \mathrm{~mW} / \mathrm{cm}^{2}$ for 20 seconds); the curing light (Bluephase G2; Ivoclar Vivadent) was calibrated at the beginning of each clinic session and irradiance was 1400 $\mathrm{mW} / \mathrm{cm}^{2}$. Final restorations were finished and polished with fluted composite finishing burs and silicone polishing cones, respectively.

\section{SELECTIVE ETCHING PLACEMENT}

Total Etch (37\% phosphoric acid; Ivoclar Vivadent) was carefully placed on the enamel margin with no intentional placement on the dentin within the lesion. The adhesive (Adhese Universal) was then applied as instructed by the manufacturer. Tetric EvoCeram composite was placed and each increment light-cured for 20 seconds. The restorations were then finished and polished in the same manner as the self-etch group.

\section{POST-PROCEDURE IMAGES AND IMPRESSIONS}

A post-operative intraoral image was obtained for each restoration using the same camera as previously mentioned and impressions were made using polyvinyl siloxane (Virtual; Ivoclar Vivadent) and poured in epoxy for SEM marginal analysis. Each 
restoration had a baseline evaluation to document retention, marginal discoloration, and marginal adaptation by two of five calibrated IUSD faculty members (Appendices 5 and 6). Since most patients were anesthetized for the restorative procedure, sensitivity was measured pre-operatively but not immediately post-operatively. The next evaluation of sensitivity took place at the six-month recall.

\section{CALIBRATION OF EVALUATORS}

Five IUSD faculty members were calibrated by evaluating cervical resin composite restorations in a separate IRB-approved calibration study that allowed enrollment of 40 subjects; the percentage of restorations with agreement among the examiners was calculated for marginal discoloration, retention, marginal adaptation, and overall clinical acceptability to ensure at least an $80 \%$ agreement among evaluators (results shown in Table II).

\section{FOLLOW-UP VISITS}

Recall examinations were conducted by two calibrated IUSD faculty members at approximately 6 and 12 months from the restorative visit. Evaluators were selected based on availability; each calibrated evaluator participated in at least $10 \%$ of the recall evaluations. Each subject's medical history was updated in Axium and the subject answered questions to assure continued eligibility in the study. Evaluation of sensitivity and modified United States Public Health Service (USPHS) criteria to include retention, 
marginal discoloration, and marginal adaptation, were recorded in the same format as the baseline evaluation by said evaluators (Appendix 6).

In addition, the two calibrated evaluators made an overall determination of clinically acceptable or not clinically acceptable at each evaluation visit. "Clinically acceptable" was defined as "a restoration that may have minor flaws (i.e., superficial staining, etc.) but shows no recurrent caries and is unlikely to cause symptoms or tissue damage within 12 months (i.e., no open margins, cracked restorations, overhangs compromising periodontal health, etc.).” Intraoral digital images and impressions were made at each recall appointment using the same camera and materials as at the baseline. The subjects were compensated with checks in amounts of $\$ 25$ and $\$ 50$, respectively, at 6- and 12-month follow-up visits, for their time and travel expenses. Restorations deemed to be not clinically acceptable within the timeframe of the study were to be replaced using normal dental school protocol at no cost to the patient.

\section{STATISTICAL CONSIDERATIONS}

The two techniques were compared for differences in sensitivity, retention, marginal discoloration, marginal adaptation, and clinical acceptability at each follow-up visit using the Cochran-Mantel-Haenszel tests for stratified, ordered categorical outcomes. The study subjects were considered the "strata" in this method, so that the comparisons properly account for the use of both techniques in each subject. This method also easily extended to having multiple teeth receiving each technique for each patient. For restorations with a dentin or enamel marginal adaptation discrepancy, the two techniques were compared for differences in dentin versus enamel discrepancy 
using Fisher's Exact tests. With a final sample size of 30 subjects, the study had $80 \%$ power to detect a $35 \%$ difference between the two treatment methods, assuming twosided tests of paired proportions and a 5\% significance level. To account for attrition, the study enrolled 33 subjects. 
RESULTS 


\section{BASELINE}

The study began on September 9, 2014 and was fully populated on April 29, 2015. Subjects for the study were selected from patients of record at Indiana University School of Dentistry Clinics who met the inclusion/exclusion criteria. A total of 33 subjects, ranging in age from 20 to 75 years, were recruited for this study. Four subjects came from the 20-39 age group, 14 from the 40-59 age group, and the remaining 15 from the 60-75 age group. The study population was basically equal in terms of the ratio of men to women (17 men, 16 women). A total of 81 lesions were identified and restorations were placed in 33 subjects. Of these 81 lesions, 62 exhibited dentin sclerosis; 46 were "wedge" or "V" shaped, and the remaining 35 were "saucer" shaped; and 44 exhibited occlusal wear facets, while the remaining 37 did not. No significant differences were found between groups in regard to these characteristics at baseline. Sensitivity was rated on a scale of $0-10$. The pre-operative data are presented in Table 2 . Thirty of the subjects requested anesthesia. Rubber dam isolation using a 212 clamp and greenstick modeling compound was used for $100 \%$ of the restorations placed. Of the 81 total restorations, 41 were placed utilizing the selective enamel etch protocol and 40 were placed with the self-etch protocol. Seventy-eight restorations received ratings of Alpha for marginal adaptation, with the remaining three receiving ratings of Bravo for pinpoint "catches" deemed to be clinically insignificant. All 81 restorations received ratings of 
Alpha for marginal discoloration and retention and all 81 restorations were rated as "clinically acceptable."

\section{6-MONTH DATA}

Six-month data collection was completed on November 5, 2015. Thirty-one subjects attended their follow-up visits; two subjects were lost to follow-up and were excluded from the study.

No significant differences were found between groups at six months. Marginal adaptation was significantly worse at six months than at baseline for Selective Etch $(p=0.0094)$, but there was no difference for Self Etch $(p=0.51)$. Marginal discoloration

did not change significantly from baseline to 6 months for either Selective Etch $(p=0.32)$ or Self Etch $(\mathrm{p}=0.16)$. Sensitivity improved from baseline to 6 months for Selective Etch $(\mathrm{p}=0.0024)$ and Self Etch $(\mathrm{p}=0.0010)$. Of 76 restorations, sensitivity improved in 27 . One restoration improved from a sensitivity of 10 to 2, and three restorations improved from 6 to 0 . No change was observed in 46 restorations, and sensitivity increased from 0 to 1 (on a 10-point analog scale) in 3 restorations. All the restorations were retained and deemed clinically acceptable.

\section{2-MONTH DATA}

The twelve-month data collection was completed on May 2, 2016. Thirty subjects attended their follow-up visits; two were lost to follow-up and one moved out of the area, thus excluding them from the study. 
No significant differences were found between groups at 12 months for sensitivity, retention, marginal discoloration, marginal adaptation, or clinical acceptability. For restorations with dentin or enamel discrepancy, there was no significant difference in the type of discrepancy $(\mathrm{p}=0.21)$.

Marginal adaptation did not change significantly from 6- to 12-months for either group (SelE $\mathrm{p}=0.39, \mathrm{SfE} \mathrm{p}=0.08$ ); however, adaptation was significantly worse at 12 months than at baseline for Selective Etch ( $\mathrm{p}=0.0455)$, but there was no difference for Self Etch $(\mathrm{p}=0.39)$. Marginal discoloration did not change significantly for the SelE group or the $\mathrm{SfE}$ group from baseline to 12 months ( $\mathrm{p}=1.00$ for both groups) or 6 - to 12 months ( $\mathrm{p}=0.32$ for both groups). Sensitivity improved from baseline to 12 months for SelE ( $p=0.0113$ ) and $\operatorname{SfE}(p=0.0128)$ but did not change significantly from 6 months to 12 months for $\operatorname{SelE}(\mathrm{p}=0.35)$ or $\operatorname{SfE}(\mathrm{p}=1.00)$. 
FIGURES AND TABLES 
TABLE I

Formulation of Adhese Universal

\section{Adhese Universal}

Solvent: $25.0 \%$

Ethanol $\left(64-17-5^{\star}\right)$

Water $\left(7732-18-5^{\star}\right)$

Monomer mixture: $67.1 \%$

HEMA (868-77-9*)

Bis-GMA (1565-94-2*)

Decandiol dimethacrylate (6701-13-9*)

Methacrylated phosphoric acid ester (85590-00-7*)

Methacrylated polyacrylic acid (9003-01-04; 106-91-2*)

Filler: $4.0 \%$

Fumed Silica $\left(7631-86-9^{*}\right)$

Initiators: $3.8 \%$

Ethyl p-Dimethylamino-benzoate (10287-53-3*)

Campherquinone (10373-78-1*)

Dimethylamino ethylmethacrylate $\left(2867-47-2^{*}\right)$

Stabilisers: $0.1 \%$

Butyl hydroxy toluene (128-37-0*)

Hydroquinone monomethylether $\left(150-76-5^{\star}\right)$

*CAS number 
TABLE II

Statistical analysis of faculty evaluator calibration

\begin{tabular}{|c|c|c|c|}
\hline CRITERIA & \% AGREEMENT & KAPPA & WT KAPPA \\
\hline Retention & 98 & 0.00 & - \\
\hline Marginal Adaptation & 80 & 0.24 & 0.28 \\
\hline Marginal Discoloration & 92 & 0.74 & 0.76 \\
\hline Clinically Acceptable & 100 & 1.00 & - \\
\hline
\end{tabular}

TABLE III

Pre-operative sensitivity rated from 0 to 10 by subjects

\begin{tabular}{|l|c|c|c|c|c|c|c|c|c|c|c|r|}
\hline Rating & 0 & 1 & 2 & 3 & 4 & 5 & 6 & 7 & 8 & 9 & 10 & Total \\
\hline Frequency & 51 & 9 & 9 & 3 & 3 & 2 & 3 & 0 & 0 & 0 & 1 & 81 \\
\hline
\end{tabular}

TABLE IV

12-month post-operative sensitivity rated from 0 to 10 by subjects

\begin{tabular}{|l|c|c|c|c|c|c|c|c|c|c|c|c|}
\hline Rating & 0 & 1 & 2 & 3 & 4 & 5 & 6 & 7 & 8 & 9 & 10 & Total \\
\hline Frequency & 62 & 9 & 1 & 1 & 0 & 0 & 1 & 0 & 0 & 0 & 0 & 74 \\
\hline
\end{tabular}




\section{TABLE V}

Baseline, 6-month, and 12-month data comparing group differences for marginal adaptation, marginal discoloration, retention, and sensitivity

\begin{tabular}{|c|c|c|c|c|}
\hline Outcome & Response & Selective Etch & Self-Etch & $\mathrm{p}$-value \\
\hline \multirow{8}{*}{ BL Sensitivity } & 0 & $23(62 \%)$ & $23(62 \%)$ & 0.38 \\
\hline & 1 & $5(14 \%)$ & $3(8 \%)$ & \\
\hline & 2 & $4(11 \%)$ & $4(11 \%)$ & \\
\hline & 3 & $1(3 \%)$ & $2(5 \%)$ & \\
\hline & 4 & $1(3 \%)$ & $2(5 \%)$ & \\
\hline & 5 & $1(3 \%)$ & $1(3 \%)$ & \\
\hline & 6 & $2(5 \%)$ & $1(3 \%)$ & \\
\hline & 10 & $0(0 \%)$ & $1(3 \%)$ & \\
\hline BL Retention & $\mathrm{A}$ & $37(100 \%)$ & $37(100 \%)$ & \\
\hline \multirow[t]{2}{*}{ BL Marginal Adaptation } & A & $37(100 \%)$ & $34(92 \%)$ & 0.0455 \\
\hline & B & $0(0 \%)$ & $3(8 \%)$ & \\
\hline BL Marginal Discoloration & A & $37(100 \%)$ & $37(100 \%)$ & \\
\hline BL Clinically Acceptable & YES & $37(100 \%)$ & $37(100 \%)$ & \\
\hline M6 Retention & A & $37(100 \%)$ & $37(100 \%)$ & \\
\hline \multirow[t]{2}{*}{ M6 Marginal Adaptation } & A & $31(84 \%)$ & $35(95 \%)$ & 0.23 \\
\hline & B & $6(16 \%)$ & $2(5 \%)$ & \\
\hline \multirow[t]{2}{*}{ M6 Type of Discrepancy } & Dentin & $5(100 \%)$ & $0(0 \%)$ & 0.0476 \\
\hline & Enamel & $0(0 \%)$ & $2(100 \%)$ & \\
\hline \multirow[t]{2}{*}{ M6 Marginal Discoloration } & A & $36(97 \%)$ & $36(97 \%)$ & 0.62 \\
\hline & B & $1(3 \%)$ & $1(3 \%)$ & \\
\hline \multirow[t]{4}{*}{ M6 Sensitivity } & 0 & $31(84 \%)$ & $32(86 \%)$ & 0.45 \\
\hline & 0.5 & $1(3 \%)$ & $0(0 \%)$ & \\
\hline & 1 & $4(11 \%)$ & $4(11 \%)$ & \\
\hline & 2 & $1(3 \%)$ & $1(3 \%)$ & \\
\hline M6 Clinically Acceptable & YES & $37(100 \%)$ & $37(100 \%)$ & \\
\hline M12 Retention & A & $37(100 \%)$ & $37(100 \%)$ & \\
\hline \multirow[t]{2}{*}{ M12 Marginal Adaptation } & A & $33(89 \%)$ & $32(86 \%)$ & 0.51 \\
\hline & B & $4(11 \%)$ & $5(14 \%)$ & \\
\hline \multirow[t]{2}{*}{ M12 Type of Discrepancy } & Dentin & $3(75 \%)$ & $1(20 \%)$ & 0.21 \\
\hline & Enamel & $1(25 \%)$ & $4(80 \%)$ & \\
\hline M12 Marginal Discoloration & $\mathrm{A}$ & $37(100 \%)$ & $37(100 \%)$ & \\
\hline \multirow[t]{5}{*}{ M12 Sensitivity } & 0 & $29(78 \%)$ & $33(89 \%)$ & 0.35 \\
\hline & 1 & $6(16 \%)$ & $3(8 \%)$ & \\
\hline & 2 & $1(3 \%)$ & $0(0 \%)$ & \\
\hline & 3 & $1(3 \%)$ & $0(0 \%)$ & \\
\hline & 6 & $0(0 \%)$ & $1(3 \%)$ & \\
\hline M12 Clinically Acceptable & YES & $37(100 \%)$ & $37(100 \%)$ & \\
\hline
\end{tabular}




\section{TABLE VI}

Marginal adaptation, marginal discoloration, retention, and sensitivity data for SelE and $\mathrm{SfE}$ groups compared at baseline, 6-months, and 12-months

\begin{tabular}{|c|c|c|c|c|c|c|c|}
\hline \multirow[b]{2}{*}{ Outcome } & \multirow[b]{2}{*}{ Response } & \multicolumn{3}{|c|}{ Selective Etch } & \multicolumn{3}{|c|}{ Self-Etch } \\
\hline & & BL & $6 \mathrm{M}$ & $12 \mathrm{M}$ & BL & $6 \mathrm{M}$ & $12 \mathrm{M}$ \\
\hline \multirow[t]{8}{*}{ Sensitivity } & $\begin{array}{l}0 \\
0.5\end{array}$ & $23(62 \%)$ & $31(84 \%)$ & $29(78 \%)$ & $23(62 \%)$ & $32(86 \%)$ & $33(89 \%)$ \\
\hline & 1 & $5(14 \%)$ & $4(11 \%)$ & $6(16 \%)$ & $3(8 \%)$ & $4(11 \%)$ & $3(8 \%)$ \\
\hline & 2 & $4(11 \%)$ & $1(3 \%)$ & $1(3 \%)$ & $4(11 \%)$ & $1(3 \%)$ & \\
\hline & 3 & $1(3 \%)$ & & $1(3 \%)$ & $2(5 \%)$ & & \\
\hline & 4 & $1(3 \%)$ & & & $2(5 \%)$ & & \\
\hline & 5 & $1(3 \%)$ & & & $1(3 \%)$ & & \\
\hline & 6 & $2(5 \%)$ & & & $1(3 \%)$ & & $1(3 \%)$ \\
\hline & 10 & & & & $1(3 \%)$ & & \\
\hline Retention & A & $37(100 \%)$ & $37(100 \%)$ & $37(100 \%)$ & $37(100 \%)$ & $37(100 \%)$ & $37(100 \%)$ \\
\hline \multirow[t]{2}{*}{ Marginal Adaptation } & A & $37(100 \%)$ & $31(84 \%)$ & $33(89 \%)$ & $34(92 \%)$ & $35(95 \%)$ & $32(86 \%)$ \\
\hline & B & $0(0 \%)$ & $6(16 \%)$ & $4(11 \%)$ & $3(8 \%)$ & $2(5 \%)$ & $5(14 \%)$ \\
\hline \multirow[t]{2}{*}{ Discrepancy } & Dentin & & $5(100 \%)$ & $3(75 \%)$ & & $0(0 \%)$ & $1(20 \%)$ \\
\hline & Enamel & & $0(0 \%)$ & $1(25 \%)$ & & $2(100 \%)$ & $4(80 \%)$ \\
\hline \multirow{2}{*}{$\begin{array}{l}\text { Marginal } \\
\text { Discoloration }\end{array}$} & & & & & & & \\
\hline & $\begin{array}{l}\text { A } \\
\text { B }\end{array}$ & $37(100 \%)$ & $\begin{array}{c}36(97 \%) \\
1(3 \%)\end{array}$ & $37(100 \%)$ & $37(100 \%)$ & $\begin{array}{c}36(97 \%) \\
1(3 \%)\end{array}$ & $37(100 \%)$ \\
\hline Clinically Acceptable & YES & $37(100 \%)$ & $37(100 \%)$ & $37(100 \%)$ & $37(100 \%)$ & $37(100 \%)$ & $37(100 \%)$ \\
\hline
\end{tabular}




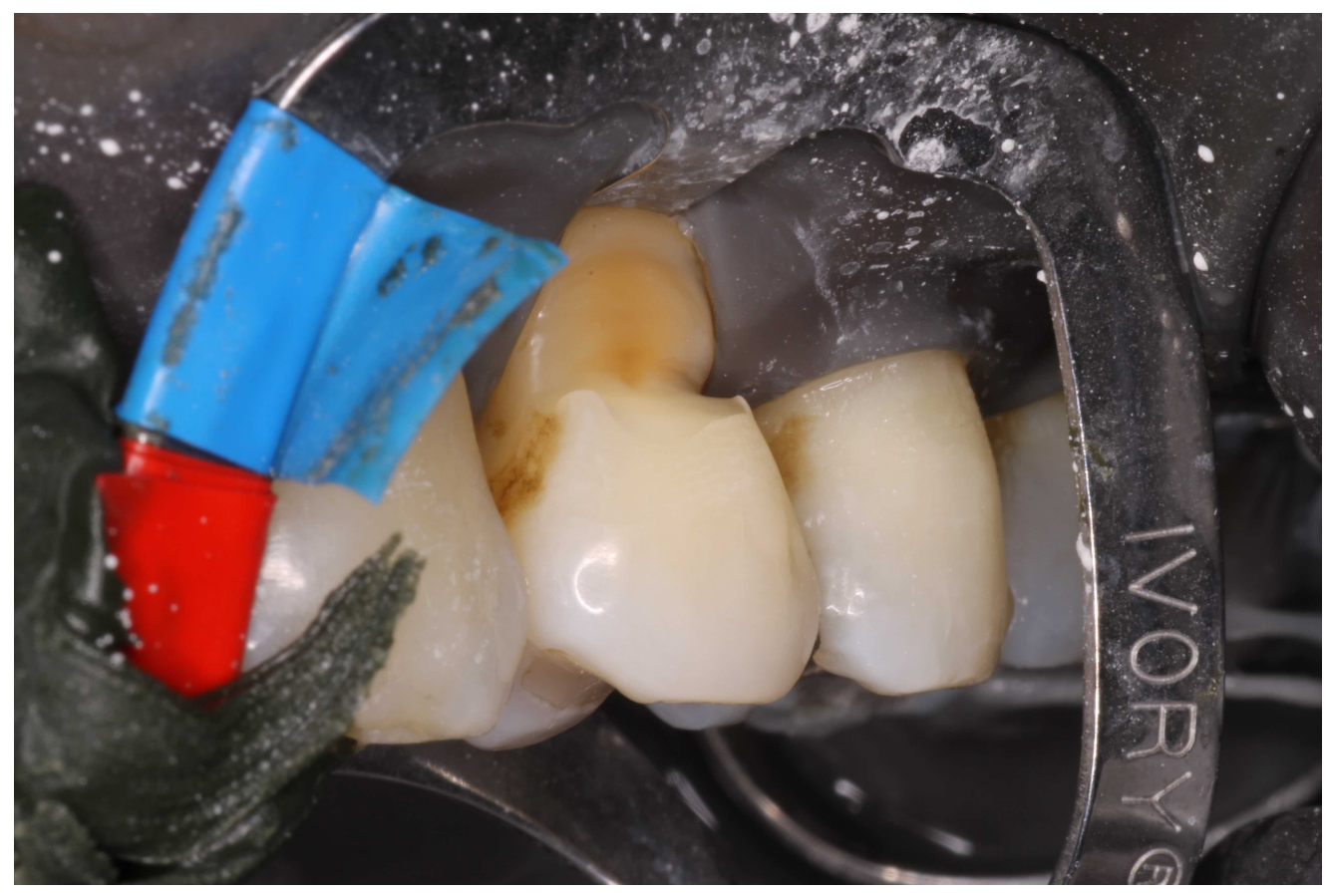

FIGURE 1. Pre-operative isolation of adjacent non-carious cervical lesion \#12-B using heavy rubber dam, W212 gingival retractor, and green stick compound.

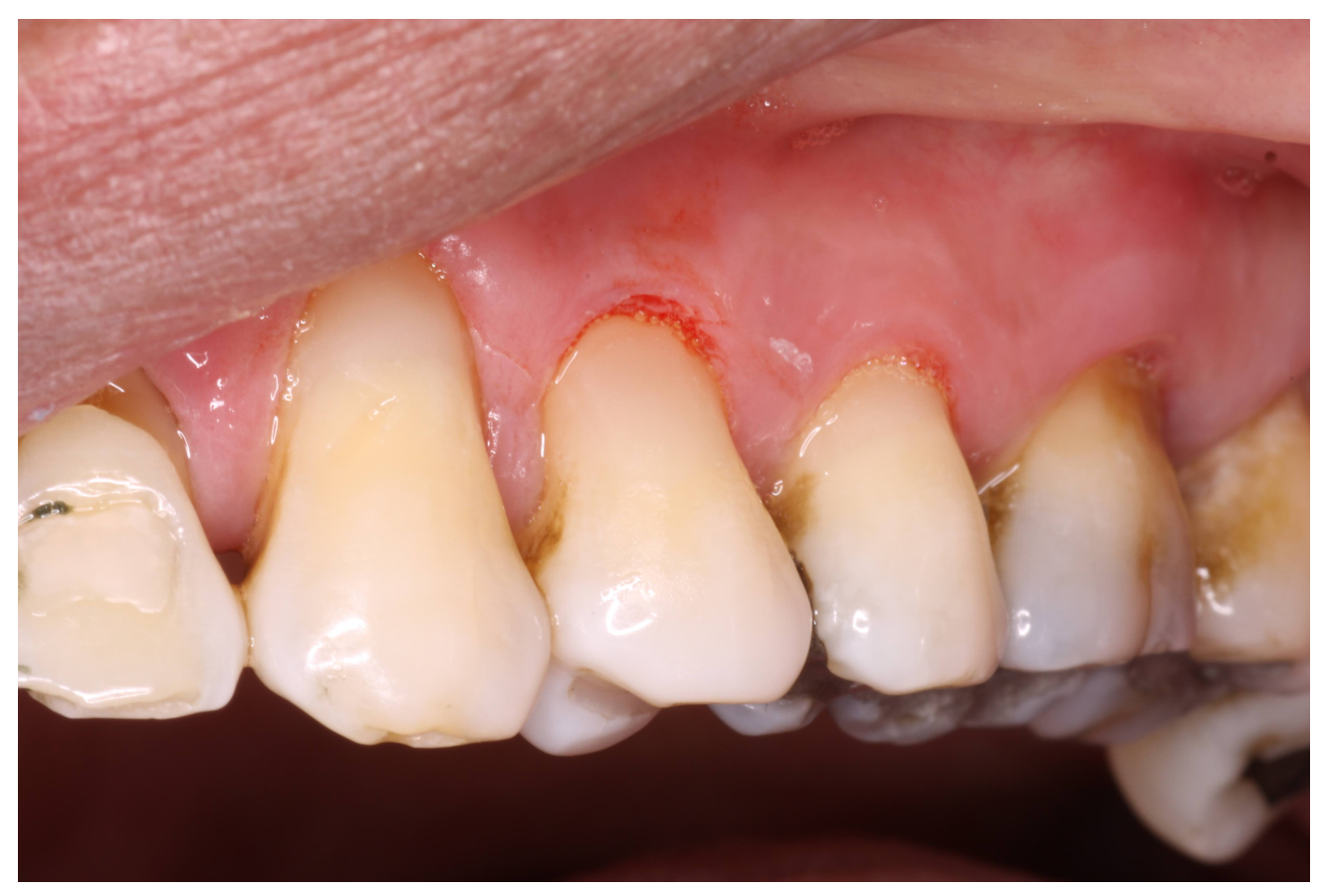

FIGURE 2. Post-operative photo of \#12-B after finishing, polishing, and removal of the rubber dam. Incremental application of Tetric EvoCeram composite was indicated due to size. 


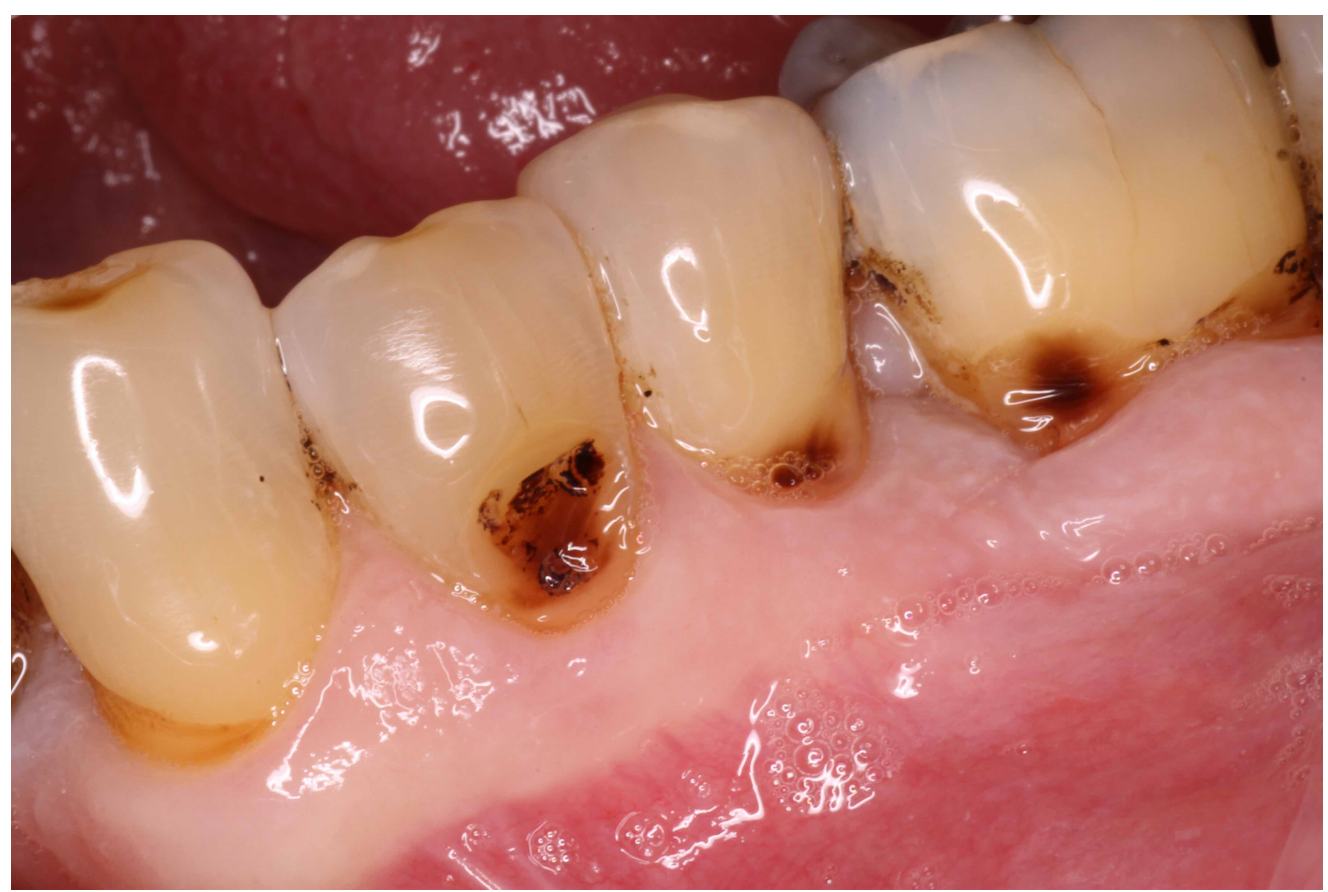

FIGURE 3. Non-carious cervical lesions \#20 and \#21 still exhibit heavy dentin staining after pre-operative pumice prophylaxis.

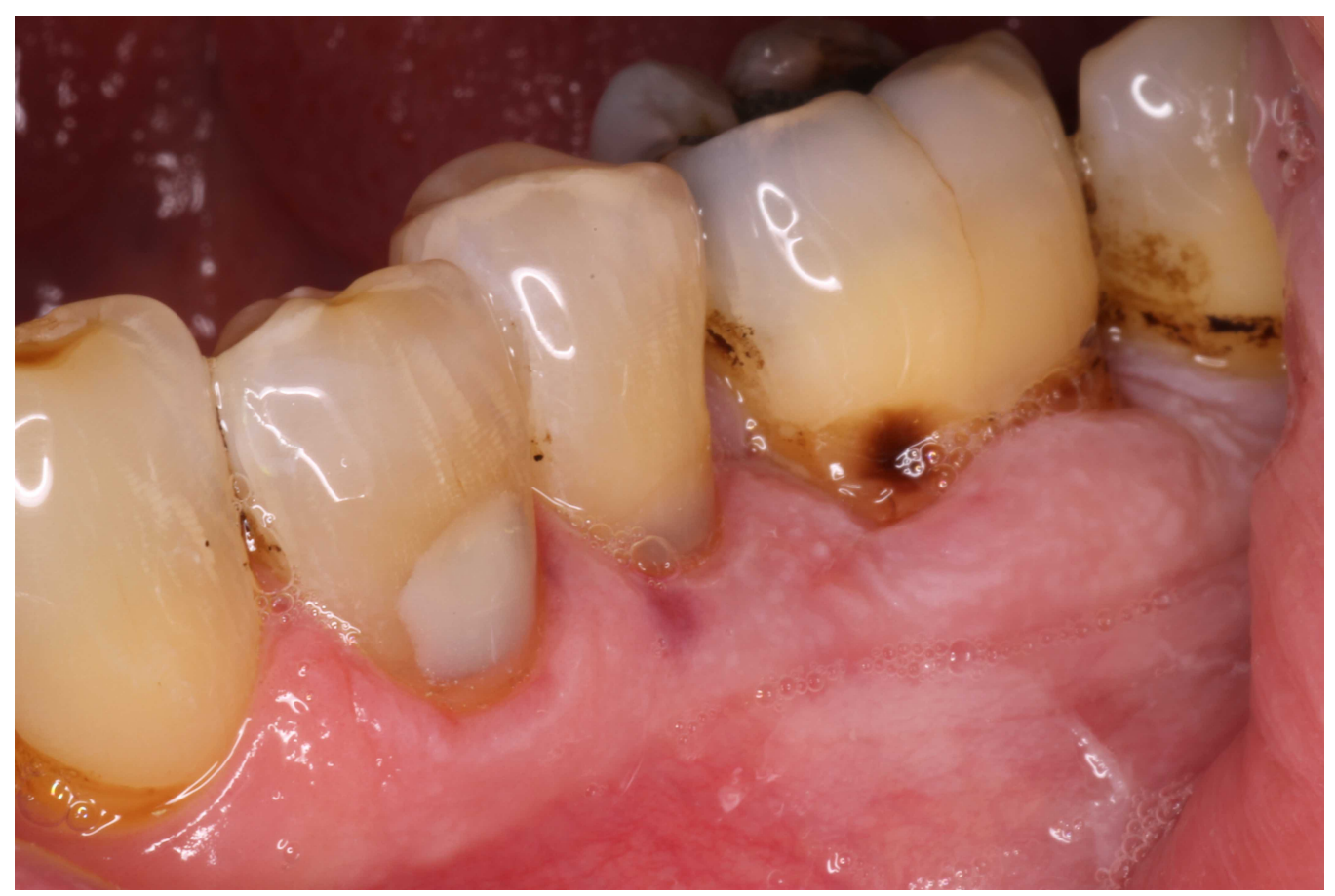

FIGURE 4. Staining still shows through both restorations \#20 and \#21 after restoration with resin composite (\#20 with Tetric EvoCeram A3, \#21 with Tetric EvoCeram A2o). Note the apparent discoloration of $\# 20$ and the unnatural opacity of \#21. These baseline photos were referred to as an aid during follow-up evaluations. 


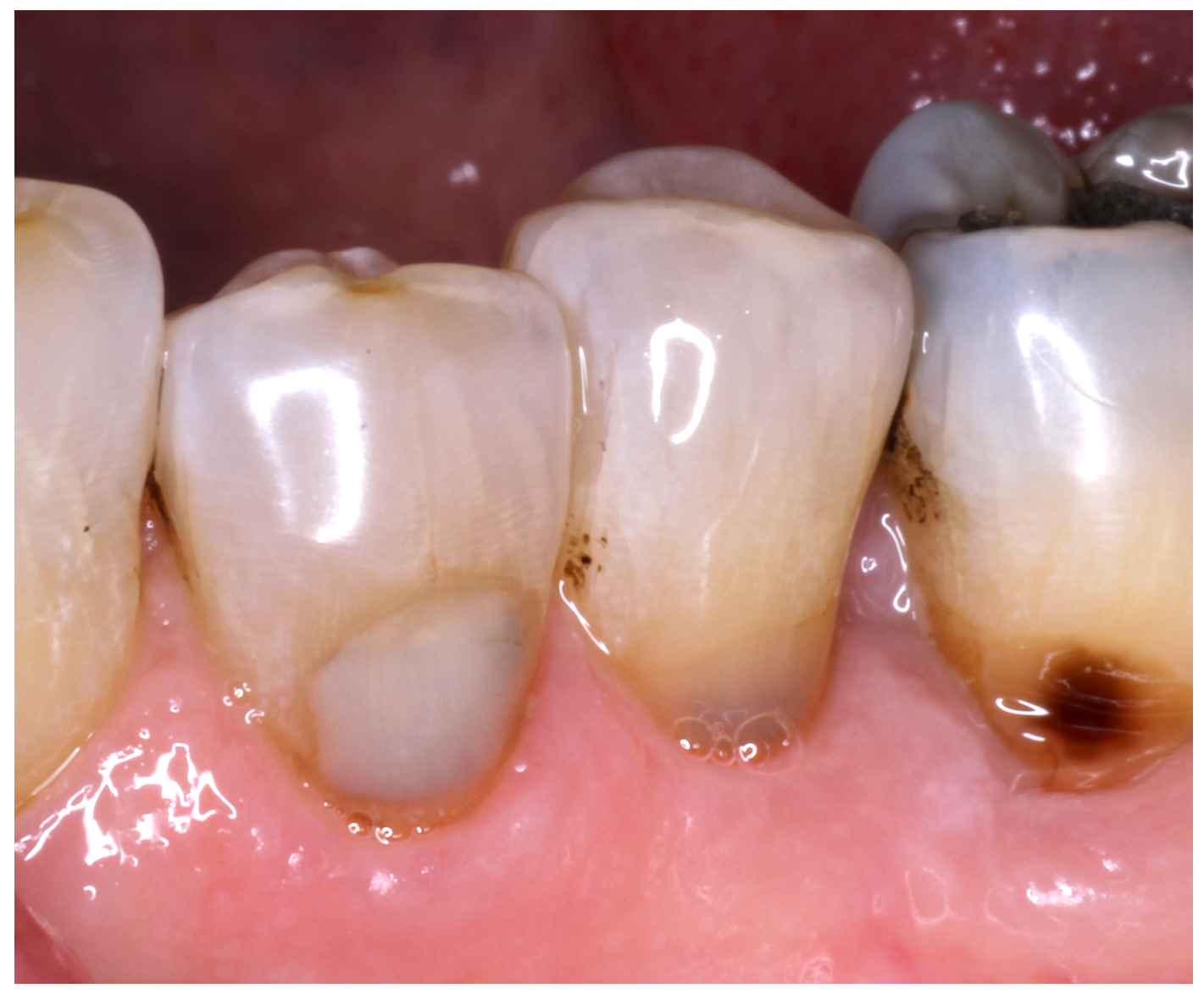

FIGURE 5. Restorations on teeth \#20 and \#21 at 6-month recall. Despite apparent discoloration, there was no change from baseline and, therefore, the restorations were scored Alpha for marginal discoloration. 
DISCUSSION 
The main finding of this randomized clinical trial was that all restorations were retained and rated as clinically acceptable one year after placement. The second major finding of this study was that, while no significant difference was found between the selfetch and selective-etch groups, a significant degradation of marginal adaptation was found in the selective-etch group between baseline and 12 months. A third observation in this study was a significant improvement in tooth sensitivity (as rated by subjects on a 010 analog scale) from pre-operative assessment to six- and 12- month recalls for both groups.

The result that all restorations were clinically acceptable 12 months after placement was the major finding of our study and was comparable to similar published studies, ${ }^{7-10}$ though only two of these studies had $100 \%$ retention at the 12 -month recall. ${ }^{7,10}$ As was the case in our study, no restorations were lost due to caries in similar studies. One reason these adhesive systems may have been so successful is that their composition is similar to sixth-generation systems that have provided reliable clinical results for more than a decade. Another reason for success, especially the lack of caries, is likely due to patient selection; these studies excluded patients with high caries risk or only included patients who were subjectively assessed by investigators to have "good oral hygiene." ${ }^{, 7}$ In fact, the very presence of non-carious cervical lesions may indicate the proclivity of patients to brush the cervical region of their dentition, thereby, actually creating abrasive lesions. 
A result which was even more interesting, though likely not as clinically relevant, was the deterioration of marginal adaptation of selective etch restorations in the current study. A similar study reported deteriorated dentin margins on two selective enamel-etch restorations (but none in the self-etch group) in their three-year data; these marginal defects were not reported in the eight-year data. ${ }^{10}$ Although no statistically significant difference was noted in the 12-month data for the current study, a trend was seen for more dentin margin defects in the SelE group and more enamel margin defects in the SfE group. One possible explanation for this marginal deterioration of the selective etch restorations could be unintentional overetching of dentin; even if phosphoric acid is placed precisely on the entire enamel margin and not on any dentin, the acid is then rinsed off with a water stream, potentially causing undesired contact with dentin before it is completely removed. Another possible explanation could be dentin desiccation resulting in diminished bonding performance. This could occur via rinsing etchant material and drying the enamel before adhesive placement in conjunction with the selective enamel etch technique; this step is not part of the self-etch technique and, therefore, dentin desiccation would be unlikely for restorations placed using a self-etch technique.

The third major finding of our study was an improvement in patient-reported sensitivity as determined by an air blast from about $1 \mathrm{~cm}$ away from the tooth surface. Numerous studies have determined that restoring non-carious cervical lesions with selfetch resin bonding is effective in reducing sensitivity, ${ }^{7}$ even when compared to topical treatment with a desensitizing dentifrice $(5 \%$ potassium nitrate toothpaste $){ }^{5}$ A simple explanation could be the presence of a physical barrier (the adhesive and restoration) 
between the tooth structure and the oral environment. Another less obvious explanation is specific to self-etch adhesive systems. Incorporation of the smear layer into the hybrid layer limits the depth of dentin etching; this limits over-etching, which some hypothesize to be a cause of post-operative sensitivity following resin bonding with total-etch systems.

Our study has many strengths, including the use of non-carious cervical lesions, utilization of a split mouth design, use of rubber dam isolation, and calibrated evaluators.

The American Dental Association recommends testing clinical effectiveness on non-carious cervical lesions for many reasons: the non-carious nature of the lesions does not require any preparation for the purpose of caries excavation, the presence of observable dentin and enamel margins is a unique advantage, and restorative treatment with either glass ionomer or resin composite is the suggested intervention in mediumlarge lesions. As discussed in the methods section, the resin composite was adapted first to enamel, instead of dentin. This is due to the superior adhesive interface between resin and enamel when compared to resin and dentin. Since the first increment placed will subsequently be subjected to more stress than the last increment placed, the enamel margin is more suited to handle this stress than the cervical dentin margin.

A split-mouth design gives a distinct advantage over other methodologies; it ensures that each patient has a restoration from each test group, which, in turn, ensures that the long list of various environmental factors (diet, oral hygiene, salivary composition and flow, etc.) have been controlled for in each patient, although no attempt was made to account for "handedness" of the participants, which may influence the amount of plaque routinely present on the right and left side of the mouth. This increases 
the likelihood that any differences seen are indeed due to the materials/techniques being studied and decreases the likelihood that these differences are attributable to uncontrolled environmental factors.

More than 150 years after its inception, the rubber dam remains the gold standard in procedural isolation, especially for procedures that require the use of moisturesensitive materials (i.e., resin adhesives). For materials used in the current study, rubber dam isolation was a recommendation found in the manufacturer's instructions for use. Interestingly, two similar studies ${ }^{7,9}$ that used only cotton-roll isolation saw significant deterioration in both marginal discoloration and marginal adaptation in the "self-etch" groups. This result was not seen in any of the similar studies ${ }^{8,10}$ in which rubber dam isolation was used, including the current study.

The calibration of evaluators gives validity to the results of any clinical trial. A pilot study was used to calibrate the five evaluators in this study; all combinations of pairs of evaluators evaluated the same restoration at least once. To ensure consistency, all restorations were placed by one operator; this operator was not one of the five calibrated evaluators.

Despite its strengths, our study has several limitations. One limitation is the technique sensitivity of the selective-etch technique; the very basis of the technique depends on the ability of the operator to precisely place phosphoric acid on the entire enamel margin while completely avoiding dentin. If phosphoric acid is not applied to the entire enamel margin, the remaining enamel will remain unetched and will theoretically have a compromised bond in the areas that are only conditioned with mild acid in the self-etch adhesive. Conversely, if too much phosphoric acid is placed and some is 
inadvertently placed on dentin, the dentin would surely be overetched and complete infiltration of the resin adhesive would be unlikely, if not impossible. This presents a problem, since the precision of placing acid-etch gel is limited. While using a viscous etching gel, rather than a liquid, gives the operator more control over where the acid goes, there is no way to ensure complete precision; while it is doubtful that an error over such a small area would have clinically-evident consequences, this has yet to be definitively determined.

Although using non-carious Class $\mathrm{V}$ lesions provides many advantages for adhesive studies, they also present many unique challenges: first, these lesions are relatively difficult to isolate compared to other lesions. Fortunately, the use of a rubber dam, 212 retainer, and green stick compound ensured isolation of the operating field, but this typically requires anesthetizing the area and any rotations/malpositioning of the dentition complicates the isolation process. Alternatively, attempting to restore these lesions (which usually extend subgingivally) without a rubber dam would severely compromise the integrity of the adhesive interface, which was, in many cases, already compromised by the presence of sclerotic dentin (discussed previously). In addition to the difficulties of isolation and dentin bonding, the presence of these lesions likely means that these areas of tooth structure have been subjected to sufficient occlusal force to cause flexure and subsequent loss of structure. Restoration of these lesions does not eliminate the occlusal force on the teeth and this force is then transferred to the adhesive interface; this can act to avulse the restoration. ${ }^{4}$

Another limitation of our study was the challenge of recruiting subjects to meet the study's prescribed criteria. An even number of subjects from each age group was 
originally desired to adjust for potential confounding. Specifically, for our study, the ideal scenario would have been to have half the subjects from each gender (which was achieved) and approximately one-third of the subjects from each age group (20-39, 4059, and 60-75). As reported in the results section, this was not the case, as only four subjects were recruited from the 20-39 age group, while the 40-59 and 60-75 age groups had 14 and 15 subjects, respectively. This is likely due to the etiology of non-carious cervical lesions; NCCLs are progressive in nature and occur gradually, over a course of decades, not months or years. With this in mind, one would not expect many patients under the age of 30 to exhibit NCCLs without profound pathological parafunction or acid reflux. This imbalance in age groups is still acceptable for the purpose of this study.

Other limitations were due to our study's protocol prohibiting mechanical preparation with burs. The only treatment of the lesions was with nonfluoridated pumice used with a prophy brush. This presented three issues: the persistence of sclerotic dentin as a bonding substrate in many cases, the lack of beveled enamel as a bonding substrate, and the persistence of stained dentin that was not always able to be blocked out adequately at restorative margins. As discussed earlier, bonding to sclerotic dentin without removing the most superficial layer with a bur, has been shown to compromise the efficacy of adhesive systems. ${ }^{29-31} \mathrm{Next}$, the lack of beveled enamel has been shown to increase microleakage and compromise the quality of the bonding interface. ${ }^{48}$ Though the manufacturer's instructions for use recommended longer etching times (up to 60 seconds) for unprepared enamel, the instructions also specified that the etch time for selective enamel etching was only to be $15-30$ seconds; therefore, 20 seconds was the time used for this study. Finally, there were a few cases in which stains were not removed 
by nonfluoridated pumice and a prophy brush. This meant that the resin composite would be placed directly over the stained dentin; but in some cases, this created the appearance of marginal discoloration at recall appointments unless opaque resin composite was used which, conversely, presented the problem of an unnatural appearance. When a body shade resin composite was used and the stain remained visible through the restoration, as depicted in Figures 3, 4, and 5, evaluators viewed preoperative and baseline placement photos and compared the appearance of the restorations at the recall visit to the baseline visit to determine if the discoloration could be attributed to the underlying dentin stain or if it was new stain due to microleakage. Discoloration due to underlying dentin still received an Alpha rating, while only discoloration due to leakage at the adhesive interface would receive a Bravo or Charlie rating. Finally, a "drift" in evaluator calibration was possible due to lack of formal recalibration during the course of the study; this did not, however, appear to be an issue. The $100 \%$ retention rate for both groups suggests that this adhesive is appropriate for placement of resin composite restorations, even on sclerotic dentin substrates. Though clinical recommendations cannot be made based on only 12 months of data, these results suggest the prospect of a class of adhesives that may simplify resin-bonding protocols while delivering clinically acceptable results. 
SUMMARY AND CONCLUSIONS 
The 12-month data shows no evidence that one method is superior to another across any of the measured criteria; this is consistent with most of the current available literature. Both groups showed acceptable results 12 months after restoration placement and either technique seems to be appropriate for the placement of resin composite restorations in non-carious cervical lesions. The statistically significant decrease in subject-reported sensitivity shows that either technique would be especially appropriate for patients with non-carious cervical lesions greater than or equal to $1 \mathrm{~mm}$ in depth who report thermal sensitivity. Retention for all restorations at 12-months was $100 \%$. 
REFERENCES 
1. Black G. A work on operative dentistry. Pathology of hard tissues of the teeth. 1 ed. Chicago, IL: Medico-Dental Publishing; 1907.

2. Miller W. Experiments and observations on the wasting of tooth tissue variously designated as erosion, abrasion, chemical abrasion, denudation, etc. Dental Cosmos 1907;49(2):109-24.

3. Grippo J0. Abfractions: a new classification of hard tissue lesions of teeth. J Esthet Dent 1991;3(1):14-9.

4. Grippo JO, Simring M, Coleman TA. Abfraction, abrasion, biocorrosion, and the enigma of noncarious cervical lesions: a 20-year perspective. J Esthet Restor Dent 2012;24(1):10-23.

5. Veitz-Keenan A, Barna JA, Strober B, et al. Treatments for hypersensitive noncarious cervical lesions: a Practitioners Engaged in Applied Research and Learning (PEARL) Network randomized clinical effectiveness study. J Am Dent Assoc 2013;144(5):495-506.

6. Hanabusa M, Mine A, Kuboki T, et al. Bonding effectiveness of a new "multimode" adhesive to enamel and dentine. J Dent 2012;40(6):475-84.

7. Can Say E, Ozel E, Yurdaguven H, Soyman M. Three-year clinical evaluation of a two-step self-etch adhesive with or without selective enamel etching in non-carious cervical sclerotic lesions. Clin Oral Investig 2014;18(5):1427-33.

8. Perdigão J, Kose C, Mena-Serrano AP, et al. A new universal simplified adhesive: 18-month clinical evaluation. Oper Dent 2014;39(2):113-27.

9. Fron H, Vergnes J-N, Moussally C, et al. Effectiveness of a new one-step selfetch adhesive in the restoration of non-carious cervical lesions: 2-Year results of a randomized controlled practice-based study. Dent Mater 2011;27(3):304-12.

10. Peumans M, De Munck J, Van Landuyt KL, et al. Eight-year clinical evaluation of a 2-step self-etch adhesive with and without selective enamel etching. Dent Mater 2010;26(12):1176-84.

11. Hashimoto M. A review: micromorphological evidence of degradation in resin-dentin bonds and potential preventional solutions. J Biomed Mater Res B Appl Biomater 2010;92(1):268-80.

12. Fejerskov T. Dental enamel. In: Mjor I, Fejerskov 0, editors. Human Oral Embryology and Histology. Copenhagen: Munksgaard; 1986. p. 5-89.

13. Shimada Y, Tagami J. Effects of regional enamel and prism orientation on resin bonding. Oper Dent 2003;28:20-27.

14. Opdam NJ, Roeters JJ, Kuijs R, Burgersdijk RC. Necessity of bevels for box only Class II composite restorations. J Prosthet Dent 1998;80(3):274-9.

15. Hilton TJ, Ferracane JL. Cavity preparation factors and microleakage of Class II composite restorations filled at intraoral temperatures. Am J Dent 1999;12(3):123-30. 
16. Robinson C, Shore R, Brookes S, et al. The chemistry of enamel caries. Crit Rev Oral Biol Med 2000;11:481-95.

17. Buonocore MG. A simple method of increasing the adhesion of acrylic filling materials to enamel surfaces. J Dent Res 1955;34(6):849-53.

18. Buonocore MG. The Use of Adhesives in Dentistry. Springfield, IL: Thomas; 1975.

19. Van Meerbeek B, De Munck J, Yoshida Y, et al. Buonocore Memorial Lecture. Adhesion to enamel and dentin: current status and future challenges. Oper Dent 2003;28(3):215-35.

20. Nanci A. Dentin-pulp complex. In: Nanci A, editor. Ten Cate's Oral Histology: Development, Structure, and Function. 6 ed. St. Louis: Mosby; 2003. p. 192239.

21. Hashimoto M, Tay FR, Svizero NR, et al. The effects of common errors on sealing ability of total-etch adhesives. Dent Mater 2006;22(6):560-68.

22. Armstrong SR, Jessop JL, Vargas MA, et al. Effects of exogenous collagenase and cholesterol esterase on the durability of the resin-dentin bond. J Adhes Dent 2006;8(3):151-60.

23. Toledano M, Osorio R, Osorio E, et al. Effect of bacterial collagenase on resindentin bonds degradation. J Mater Sci Mater Med 2007;18(12):2355-61.

24. Abdalla AI, Feilzer AJ. Four-year water degradation of a total-etch and two self-etching adhesives bonded to dentin. J Dent 2008;36(8):611-17.

25. Hilton TJ, Ferracane JL, Broome JC. Summitt's fundamentals of operative dentistry: a contemporary approach. Fourth ed. Hanover Park, IL: Quintessence Publishing Co. Inc; 2013.

26. Walter C, Kress E, Gotz H, et al. The anatomy of non-carious cervical lesions. Clin Oral Investig 2014;18(1):139-46.

27. Peumans M, De Munck J, Van Landuyt K, Lambrechts P, Van Meerbeek B. Five-year clinical effectiveness of a two-step self-etching adhesive. J Adhes Dent 2007;9(1):7-10.

28. Van Landuyt KL, De Munck J, Ermis RB, Peumans M, Van Meerbeek B. Fiveyear clinical performance of a HEMA-free one-step self-etch adhesive in noncarious cervical lesions. Clin Oral Investig 2014;18(4):1045-52.

29. Tay FR, Pashley DH. Resin bonding to cervical sclerotic dentin: a review. J Dent 2004;32(3):173-96.

30. Kwong SM, Cheung GS, Kei LH, et al. Micro-tensile bond strengths to sclerotic dentin using a self-etching and a total-etching technique. Dent Mater 2002;18(5):359-69.

31. Kwong S-M, Tay FR, Yip H-K, Kei L-H, Pashley DH. An ultrastructural study of the application of dentine adhesives to acid-conditioned sclerotic dentine. J Dent 2000;28(7):515-28.

32. Chang JC, Hurst TL, Hart DA, Estey AW. 4-META use in dentistry: A literature review. J Prosthet Dent 2002;87(2):216-24.

33. Cochran MA. Lecture: Resin Adhesives in Dentistry: Indiana University School of Dentistry; 2013.

34. Kakar S, Goswami M, Kanase A. Dentin bonding agents I: complete classification-a review. World Journal of Dentistry 2011;2(4):367-70. 
35. Roberson T, Heymann H, Swift E. Art and science of operative dentistry. 5 ed; 2006.

36. Breschi L, Mazzoni A, Ruggeri A, et al. Dental adhesion review: aging and stability of the bonded interface. Dent Mater 2008;24(1):90-101.

37. Van Meerbeek B, Yoshihara K, Yoshida Y, et al. State of the art of self-etch adhesives. Dent Mater 2011;27(1):17-28.

38. Wang Y, Spencer P. Continuing etching of an all-in-one adhesive in wet dentin tubules. J Dent Res 2005;84(4):350-54.

39. Kadoma Y. Surface treatment agent for dental metals using a thiirane monomer and a phosphoric acid monomer. Dent Mater J 2002;21(2):156-69.

40. Erickson RL, Barkmeier WW, Kimmes NS. Bond strength of self-etch adhesives to pre-etched enamel. Dent Mater 2009;25(10):1187-94.

41. Sideridou I, Tserki V, Papanastasiou G. Study of water sorption, solubility, and modulus of elasticity of light-cured dimethacylate-based dental resins. Biomaterials 2003(24):655-65.

42. Salz U, Mucke A, Zimmermann J, Tay F, Pashley D. pKa value and buffering capacity of acidic monomers commonly used in self-etching primers. J Adhes Dent 2006(8):143-50.

43. Chen L, Suh BI, Brown D, Chen X. Bonding of primed zirconia ceramics: evidence of chemical bonding and improved bond strengths. Am J Dent 2012;25(2):103-08.

44. Ferracane JL. Buonocore Lecture. Placing dental composites: a stressful experience. Oper Dent 2008(33):247-57.

45. Miessler G. Inorganic Chemistry. 2nd ed: Prentice Hall; 1991.

46. Van Landuyt KL, Snauwaert J, De Munck J, et al. Systematic review of the chemical composition of contemporary dental adhesives. Biomaterials 2007;28(26):3757-85.

47. Sharma D, McGuire JA, Gallob JT, Amini P. Randomised clinical efficacy trial of potassium oxalate mouthrinse in relieving dentinal sensitivity. J Dent 2013;41 Suppl 4:S40-48.

48. Kubo S, Yokota H, Yokota H, Hayashi Y. Challenges to the clinical placement and evaluation of adhesively-bonded, cervical composite restorations. Dent Mater 2013;29(1):10-27. 


\section{APPENDICES}


APPENDIX 1. Inclusion and exclusion criteria

\section{INCLUSION CRITERIA}

1. Willing to provide written consent and authorization for participation.

2. Were between 20 and 75 years of age at the time of recruitment.

3. Had at least two non-carious cervical lesions present in canine or premolar teeth.

4. Anticipated availability for recalls (roughly 6 months, 12 months, and 24 months) through the two-year study period.

5. The lesions selected were at least $1 \mathrm{~mm}$ in depth (measured with a perio probe) and contained both enamel and dentin margins.

\section{EXCLUSION CRITERIA}

1. Severe medical complications (organ transplants, cancer, immunocompromised, long-term antibiotic or steroid therapy).

2. Active caries on study teeth.

3. Bleeding on probing of study teeth.

4. Generalized severe periodontitis.

5. Patient reported symptoms (burning mouth, loss or diminished taste, saliva amount too little, needs liquids to eat dry foods) or clinical signs (erythematous tongue, chelitis, lack of pooled saliva) associated with dry mouth.

6. Patients determined to be at a high risk for caries as determined by a Caries Risk Assessment. 


\section{APPENDIX 2. IRB Approved phone screening}

Hello, this is from the Indiana University School of Dentistry. I'm calling about a new

dental study that involves persons who need gumline fillings. Would you like to hear more about the study?

Weak areas of tooth structure near the gumline can arise from different causes. When tooth structure in these areas is lost, it can result in loss of strength, less desirable appearance, and sensitivity. For these reasons, it is recommended that dental fillings be placed in these areas to protect and restore the tooth. White "resin" fillings are usually the recommended fillings for these areas and they are bonded (held) in place using adhesive (glue-like) systems with "etching" techniques that help the bonding agents work better. The purpose of this study is to use a new adhesive system, with and without etching, to see how effective it is in restoring lost tooth structure at the gumline.

If you are interested in participating, I will review and update your medical records, ask questions to determine if you qualify (like your age and time availability), and perform an exam of your mouth. You need to have at least two teeth that need gumline fillings but do not currently have no dental decay. We will thoroughly clean the teeth, and pictures of the study teeth will be taken. Standard- of- care procedures (procedures that are normally done in a filling procedure and are not related to research) will be performed to prepare your teeth for the filling placement. At the point in the dental procedure where the etching, adhesive and filling placement occurs, I will follow the manufacturer's guidelines for placing the study adhesive and study etching, if applicable. After the study materials are applied, the fillings will be placed according to standard- of- care procedures for gumline fillings. Pictures of the teeth with these fillings in place will be taken. An impression (mold) of the teeth with the fillings will be made with a playdough- like material. You will be asked to attend three follow- up study visits at about six, 12, and 24 months from the screening/filling visit.

The purpose, procedures, risks, payments, and benefits of the study will be explained in detail during the first study visit and you will be given the chance to ask questions before deciding whether or not to participate. If you qualify and participate, you will receive your study teeth gumline fillings free and will receive $\$ 25, \$ 50$, and then $\$ 75$ for each of the three follow- up visits.

Does this sound like a study you would like to learn more about by scheduling the consenting visit?

I need to ask you a few questions before continuing. Please be advised that you need only answer yes or no to each question. I do not need details. I will not be recording any information.

1) Are you between 20 and 75 years old? YES

2) Do you suffer from dry mouth? NO

3) Do you anticipate living in this area for the next two years? YES

\section{Qualified? YES / NO}

$N O=$ I'm sorry, you do not qualify for this study. Thank you for your time. Interview Completed. $Y E S=$ It appears that you may qualify for this study.

Make an appointment for screening 
APPENDIX 3. Preoperative assessment table

\begin{tabular}{|l|l|l|l|l|l|}
\hline & $\#$ & Sclerosis & $\begin{array}{l}\text { Lesion } \\
\text { Morphology }\end{array}$ & $\begin{array}{l}\text { Occlusal } \\
\text { Facets }\end{array}$ & Pre-Op Sensitivity \\
\hline Self-Etch Tooth & & & & & \\
\hline Selective etch Tooth & & & & & \\
\hline Tooth \# ( & & & & & \\
\hline
\end{tabular}

\begin{tabular}{|ll|}
\hline Legend: & \\
Sclerosis: & $0=$ no sclerosis \\
& $1=<50 \%$ sclerotic \\
& $2=\geq 50 \%$ sclerotic \\
Lesion Morphology & S= saucer shape \\
& V= "V" shape \\
Occlusal Facets & Yes \\
& No \\
Pre-Op Sensitivity & $0-10$ \\
\hline
\end{tabular}

APPENDIX 4. Numeric pain scale

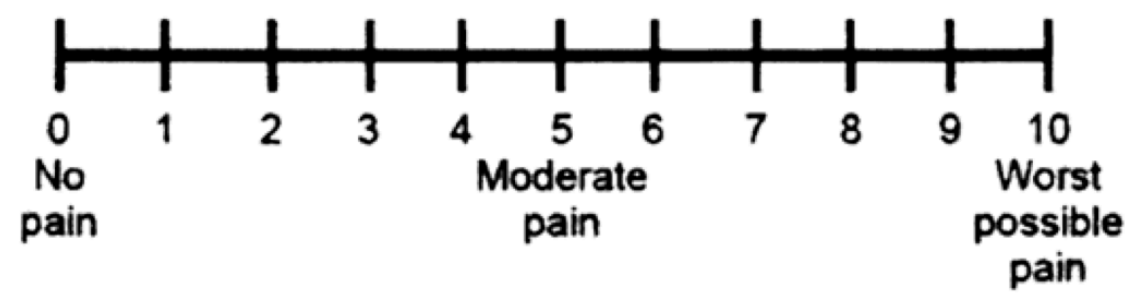


APPENDIX 5. Postoperative assessment form

\begin{tabular}{|c|c|c|c|c|c|c|}
\hline Baseline & $\#$ & Retention & Marginal Adapt. & Marg. Discoloration & Sensitivity $(0-10)$ & Clinically Acceptable? \\
\hline Tooth \# ( & & & & & & \\
\hline Tooth \# ( & & & & & & \\
\hline Tooth \# ( & & & & & & \\
\hline
\end{tabular}

\section{APPENDIX 6. Modified USPHS criteria}

Marginal Discoloration

A: There is no discoloration between the tooth and the restoration

$\mathrm{B}$ : Discoloration is present without axial penetration

$\mathrm{C}$ : Discoloration is present with axial penetration

Retention
A: Present
B: Partial loss of restoration
$\mathrm{C}$ : Absent/complete loss of restoration
Marginal Adaptation

A: Excellent continuity at resin-enamel interface; explorer exhibits no catch or one-way catch when drawn across margin

B: Explorer exhibits a two-way catch, indicating a crevice, when drawn across margin

$\mathrm{C}$ : Marginal crevice present; exposes base or dentin 

IN NON-CARIOUS CERVICAL LESIONS

by

Matthew A. Rouse

Indiana University School of Dentistry

Indianapolis, Indiana 
The "total-etch" or "etch-and-rinse" systems have been the gold standard of dental bonding for decades. However, these systems are very technique-sensitive and time-consuming compared to newer "self-etch" or "self-adhesive" systems and have been implicated in cases of postoperative sensitivity. The purpose of this study was to compare the effects of two surface treatment protocols (self-etch vs. selective-etch) on the clinical performance of a universal adhesive and resin composite in Class V noncarious cervical lesions (NCCLs).

Thirty-three volunteer subjects ( 17 male; 16 female; age range $=20$ to 75 years) having at least two NCCLs were selected from patients of record at Indiana University School of Dentistry. Each subject received one resin composite restoration (Tetric EvoCeram, Ivoclar Vivadent) utilizing a self-etch (SfE) universal adhesive (Adhese Universal, Ivoclar Vivadent) with no separate enamel etching and another restoration utilizing adhesive and selective enamel etching (SelE) with 37\% phosphoric acid $\left(\mathrm{H}_{3} \mathrm{PO}_{4}\right)$. Both the adhesive and composite were placed following the manufacturer's instructions. The two techniques were compared for differences in sensitivity, retention, marginal discoloration, marginal adaptation, and clinical acceptability at baseline and 6 months using the Cochran-Mantel-Haenszel tests for stratified, ordered categorical outcomes.

Seventy-four restorations (37 SfE, $37 \mathrm{SelE}$ ) in 30 volunteers were evaluated at 12 months. No significant differences were found between the SfE and SelE groups for 
any variable at the 12 -month recall $(\mathrm{p} \geq 0.21)$. Retention was $100 \%$ at 12 months for both groups. Marginal adaptation was significantly worse at 12 months than at baseline for $\operatorname{SelE}(\mathrm{p}=0.0163)$, but there was no difference for $\mathrm{SfE}(\mathrm{p}=0.08)$. Sensitivity improved significantly from baseline to 12 months for both SelE ( $p=0.0113)$ and SfE $(\mathrm{p}=0.0128)$.

The results obtained from this study are comparable to results observed in similar studies. Like similar studies involving self-etch adhesives in non-carious cervical lesions, our study showed no restorations lost to caries and excellent retention. The deterioration of selective-etch dentin margins was a result that differed from similar studies. A likely explanation for this finding would be the difficulty of controlling precise placement of phosphoric acid gel, causing undesired etching of dentin; this could result in suboptimal bonding to dentin.

This report on 12-month data for a two-year study indicates significantly reduced sensitivity for both the SelE and SfE groups, and deterioration of SelE marginal adaptation. No decreases in retention, marginal discoloration, or clinical acceptability were observed in either group. 



\section{CURRICULUM VITAE}

\section{Matthew A. Rouse}

September 7, 1985

May 2007

May 2011

July 2011 to July 2012

Aug 2012 to June 2013

July 2013 to June 2016
Born in Hagerstown, MD

B.A.

McDaniel College

Westminster, MD

D.D.S.

Baltimore College of Dental

Surgery, Baltimore, MD

Advanced Education in General Dentistry Residency

2D Den Bn/Naval Dental Center, Camp Lejeune, NC

Assistant Dental Officer, French Creek Branch Dental Clinic, Camp Lejeune, NC

Operative and Preventive Dentistry Indiana University School of Dentistry, Indianapolis, IN

Professional Organizations

American Dental Association

Academy of Operative Dentistry

American Academy of Dental Research

American Dental Educators Association 